\title{
Rapid simultaneous determination of cascade metabolites of acrylamide in urine for toxicokinetic profiles and short-term dietary internal exposure
}

Yiju Zhang,,${ }^{\dagger, \#}$ Qiao Wang,,${ }^{\dagger, \#}$ Wei Jia,${ }^{\dagger}$ Jun Cheng, ${ }^{\dagger}$ Li Zhu,,${ }^{\dagger}$ Yiping Ren,, Yu Zhang*,†

${ }^{\dagger}$ National Engineering Laboratory of Intelligent Food Technology and Equipment, Zhejiang Key Laboratory for Agro-Food Processing; College of Biosystems Engineering and Food Science, Zhejiang University, Hangzhou 310058, China

¥ Yangtze Delta Region Institute of Tsinghua University, Jiaxing 314006, China

\section{Supporting Information}

Figure S1. The chemical structures of acrylamide- $d_{3}$, glycidamide- ${ }^{13} \mathrm{C}_{3}$, AAMA- $d_{3}$, AAMA-sul- $d_{3}$, GAMA- $d_{3}$ and iso-GAMA- $d_{3}$. .2

Figure S2. The product ion scan mass spectrograms of 6 cascade metabolites of acrylamide. (A) acrylamide; (B) glycidamide; (C) AAMA; (D) AAMA-sul; (E) isoGAMA; (F) GAMA. .4

Figure S3. The product ion scan mass spectrograms of the isotope-labelled internal standards for 6 cascade metabolites of acrylamide. (A') acrylamide- $d_{3}$; (B') glycidamide- ${ }^{13} \mathrm{C}_{3}$; (C') AAMA- $d_{3}$; (D') AAMA-sul- $d_{3}$; (E') iso-GAMA- $d_{3}$; (F') GAMA- $d_{3}$ 
<smiles>[2H]C([2H])=C(P)C(N)=O</smiles>

$\mathrm{AA}-d_{3}$<smiles>[2H]C([2H])([2H])C(=O)N[C@H](CSCCC(N)=O)C(=O)O</smiles>

$\mathrm{AAMA}_{3}$<smiles>[2H]C([2H])([2H])C(=O)N[C@@H](CSCC(O)C(N)=O)C(=O)O</smiles>

GAMA- $d_{3}$<smiles>NC(=O)C1CO1</smiles>

$\mathrm{GA}-{ }^{13} \mathrm{C}_{3}$<smiles>[2H]C([2H])([2H])C(=O)N[C@@H](CS(=O)CCC(N)=O)C(=O)O</smiles>

AAMA-sul- $d_{3}$<smiles>[2H]C([2H])([2H])C(=O)N[C@@H](CSC(CO)C(N)=O)C(=O)O</smiles>

iso-GAMA- $d_{3}$

Figure S1. The chemical structures of acrylamide- $d_{3}$, glycidamide- ${ }^{13} \mathrm{C}_{3}$, AAMA- $d_{3}$, AAMA-sul- $d_{3}$, GAMA- $d_{3}$ and iso-GAMA- $d_{3}$. AA, acrylamide; GA, glycidamide. 
(A)

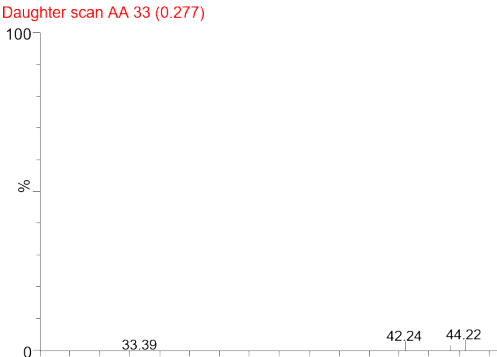

Daughters of
71.93
$1.62 \mathrm{ES}$

54.94

(B)

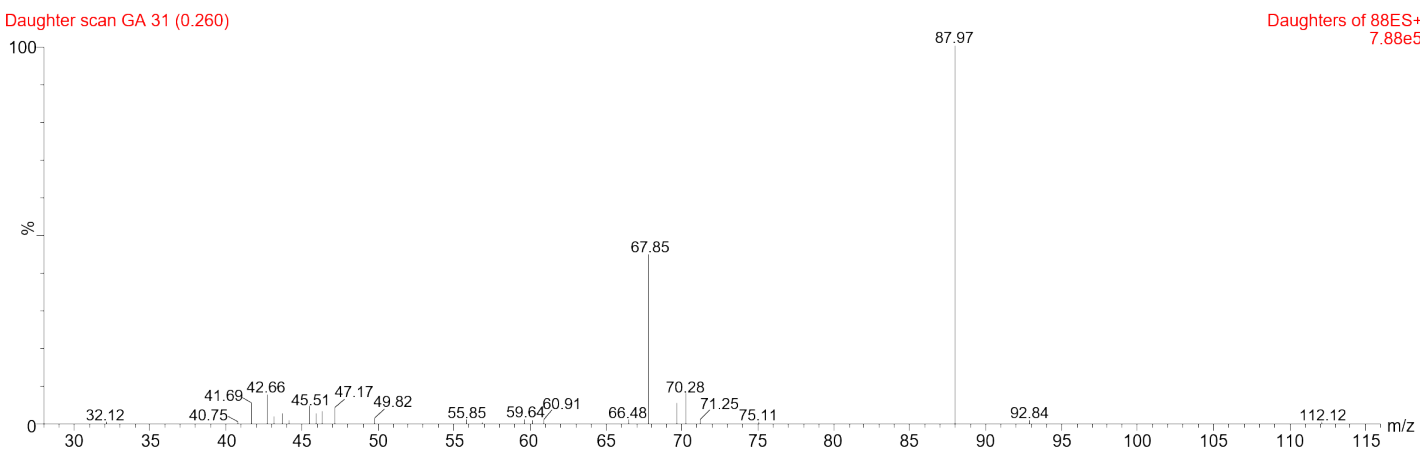

(C)

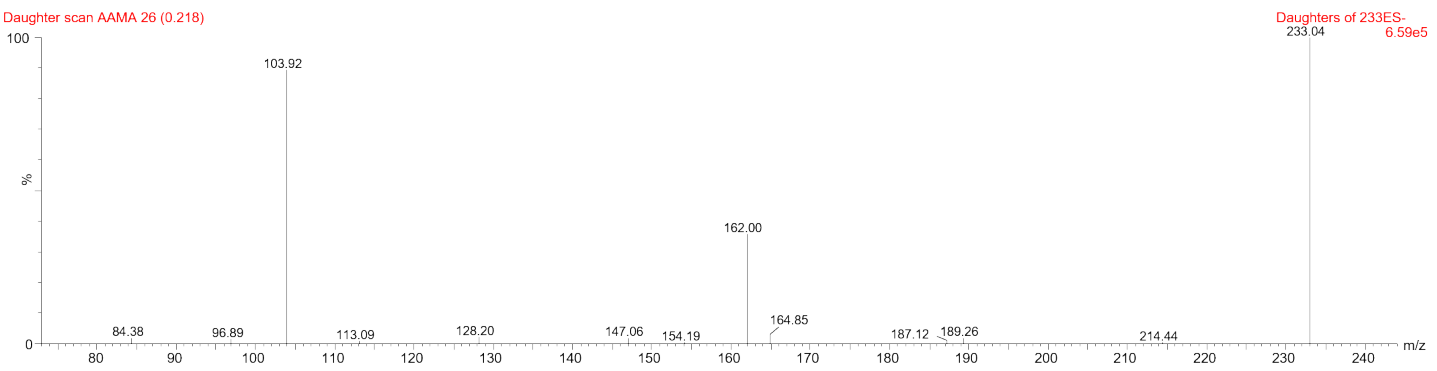

(D)

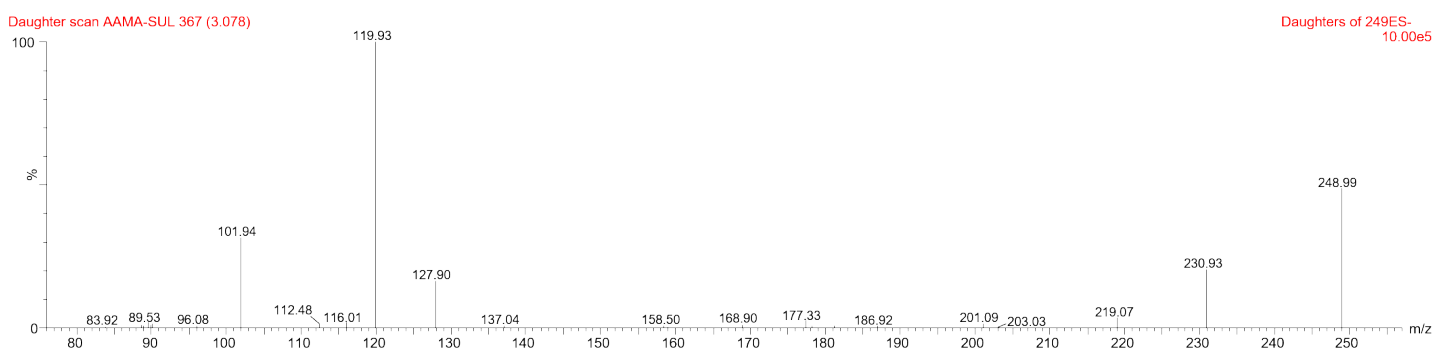


(E)

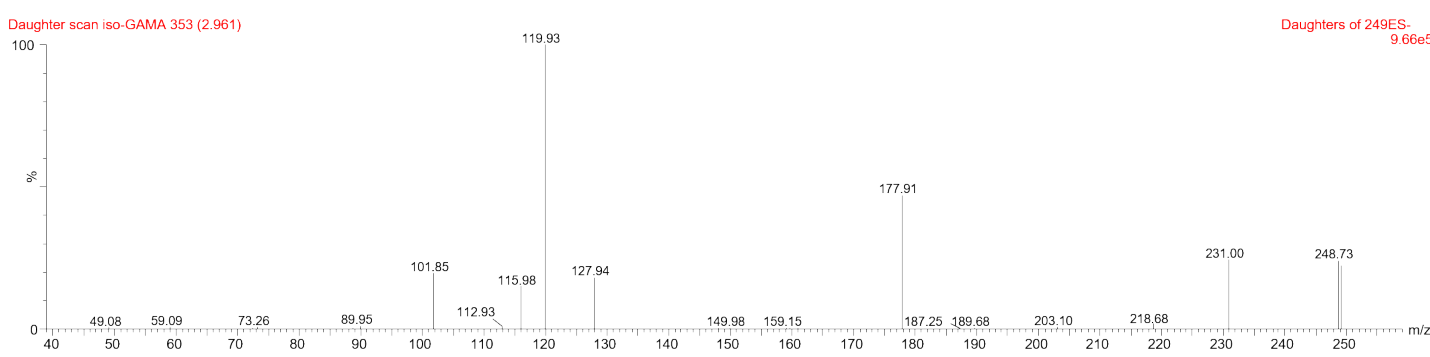

(F)

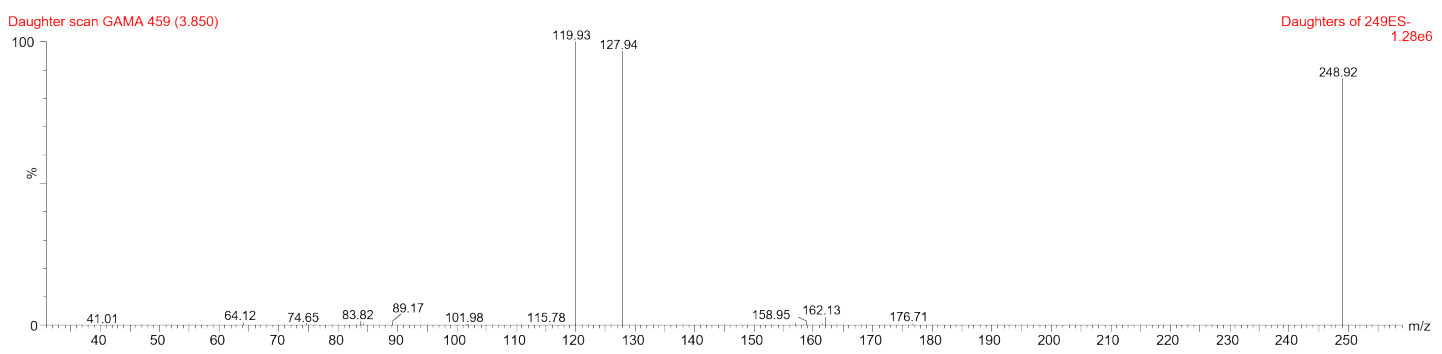

Figure S2. The product ion scan mass spectrograms of 6 cascade metabolites of acrylamide. (A) acrylamide; (B) glycidamide; (C) AAMA; (D) AAMA-sul; (E) isoGAMA; (F) GAMA. 
(A')

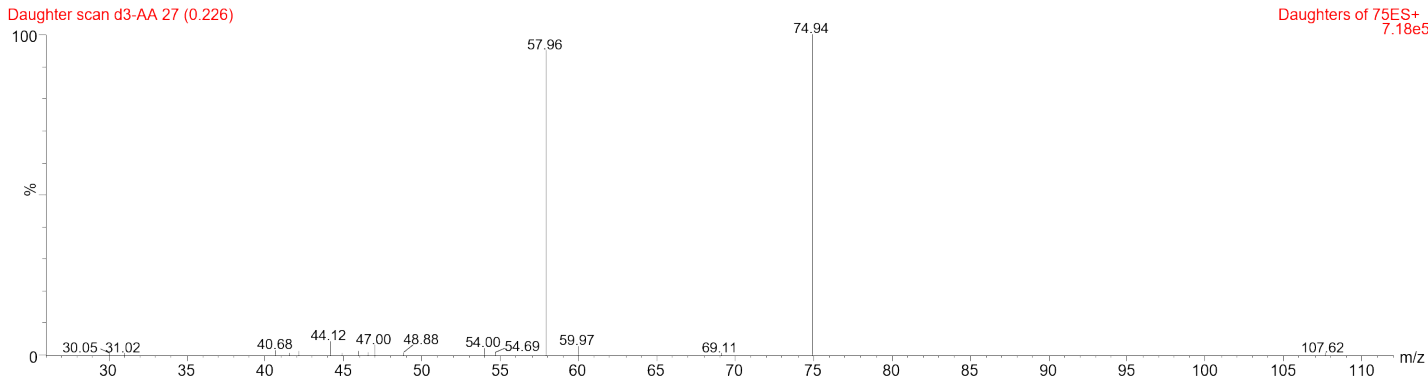

\section{(B')}

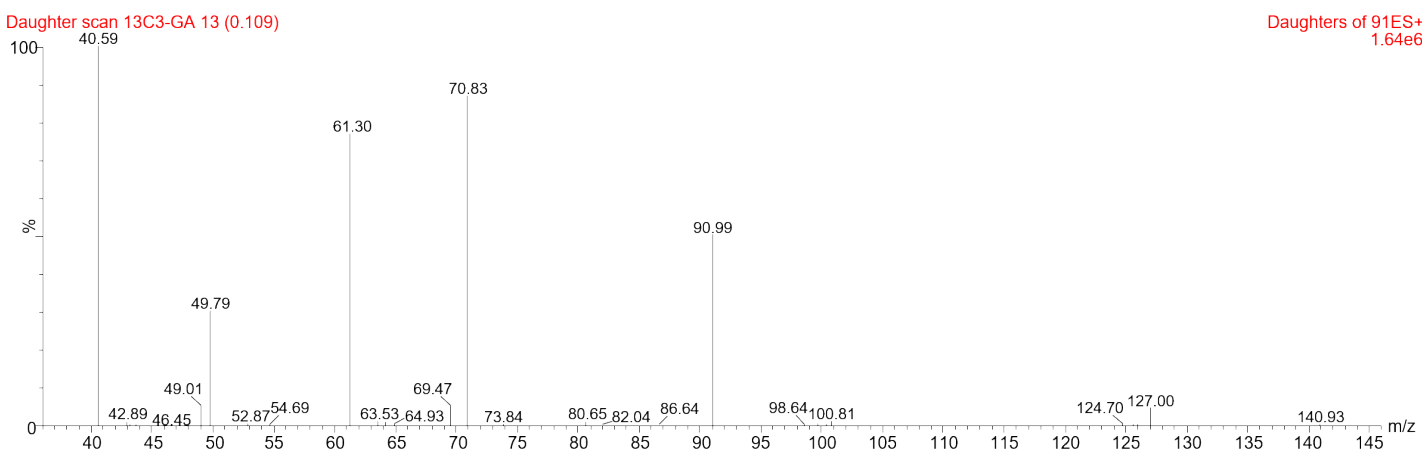

\section{(C')}

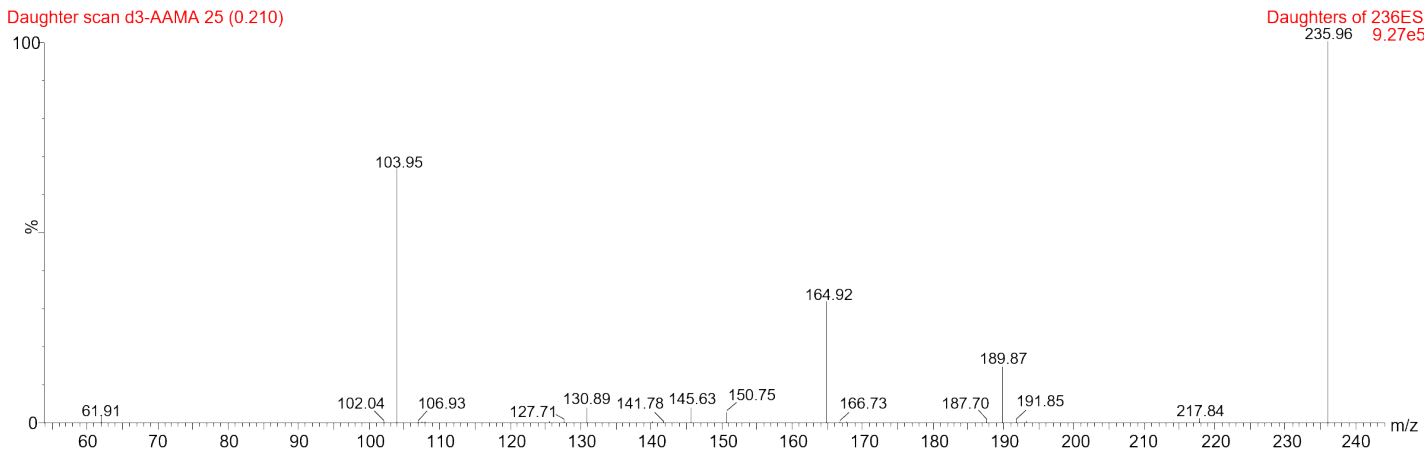

(D')

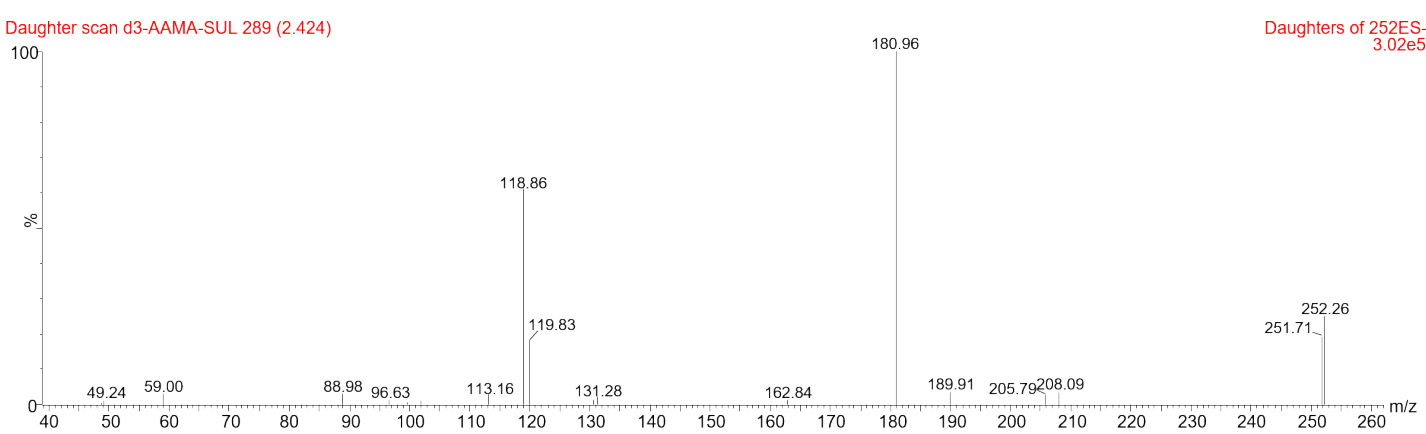


$\left(\mathbf{E}^{\prime}\right)$

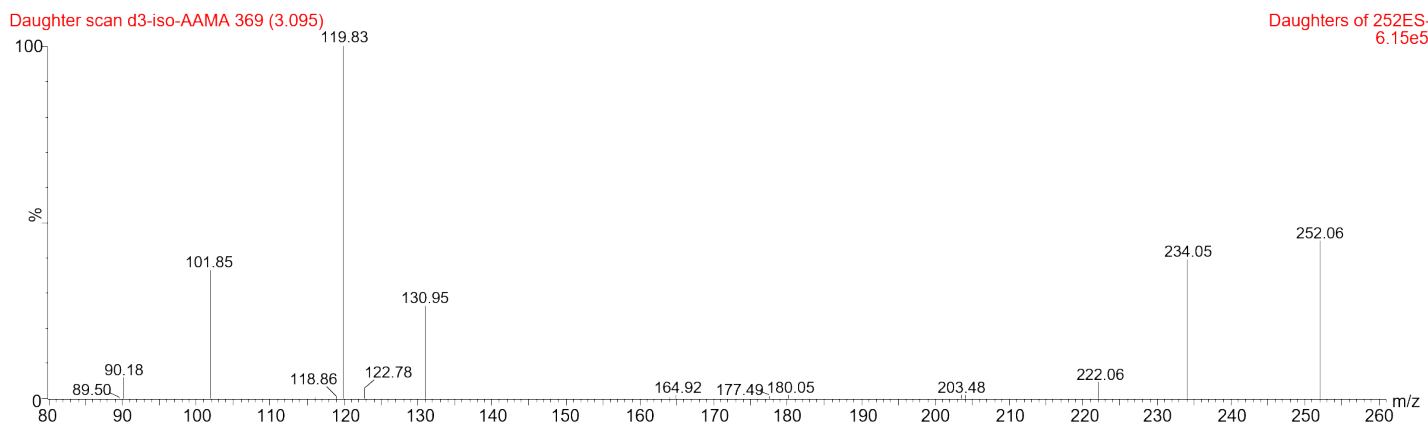

( $\left.\mathbf{F}^{\prime}\right)$

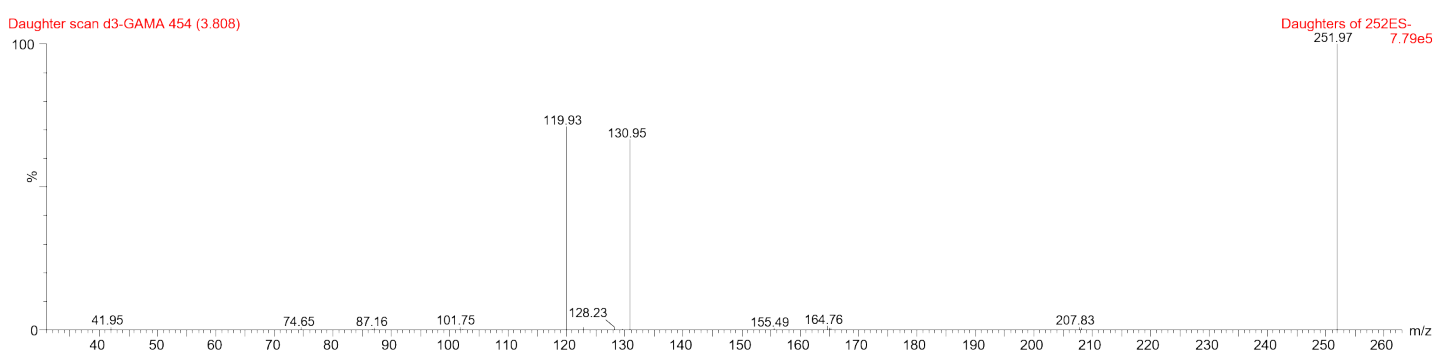

Figure S3. The product ion scan mass spectrograms of the isotope-labelled internal standards for 6 cascade metabolites of acrylamide. (A') acrylamide- $d_{3}$; (B') glycidamide- ${ }^{13} \mathrm{C}_{3}$; (C') AAMA- $d_{3}$; (D') AAMA-sul- $d_{3}$; (E') iso-GAMA- $d_{3}$; (F') GAMA- $d_{3}$. 